\title{
Amide-to-amide hydrogen bonds in the presence of pyridine functionality: Crystal structures of bis(pyridinecarboxamido) alkanes
}

\author{
Madhushree Sarkar and Kumar Biradha* \\ Department of Chemistry, Indian Institute of Technology, Kharagpur-721302, India
}

Fax:+91-3222-282252; Tel: +91-3222-283346;

E-mail: kbiradha@chem.iitkgp.ernet.in

Supporting information (31 pages)

(ORTEP drawings and Crystallographic tables for $\mathbf{2 a - 2 d}$ and $\mathbf{3 b} \mathbf{b}-\mathbf{3 e}$ ) 


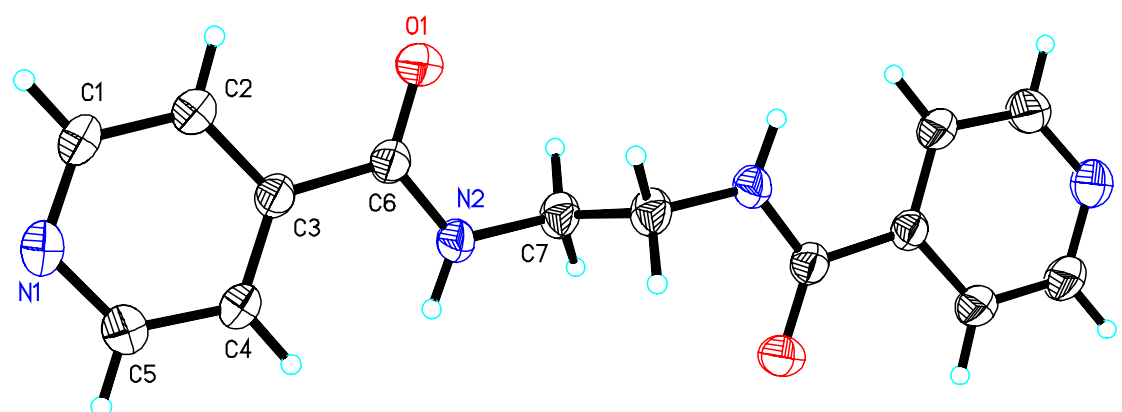

ORTEP drawing for $\mathbf{2 a}$

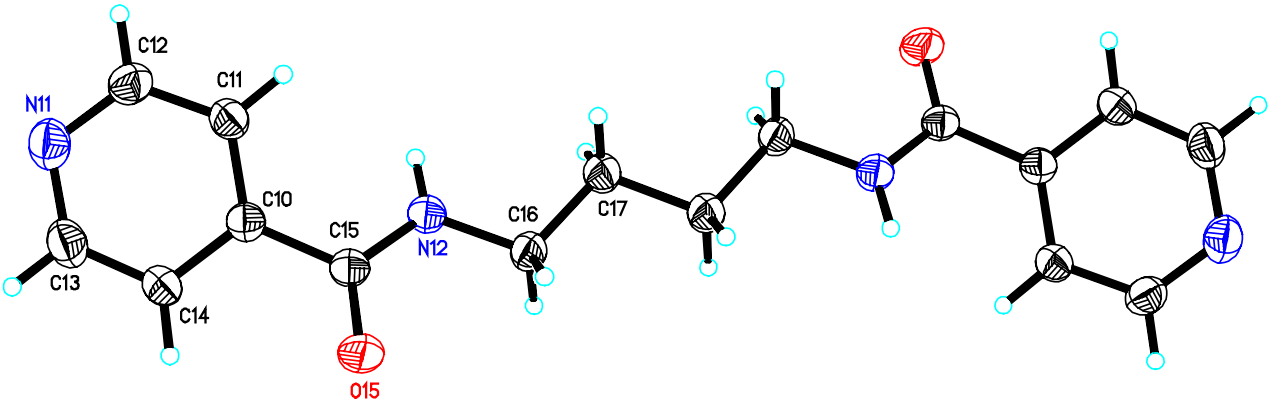

ORTEP drawing for $\mathbf{2 b}$

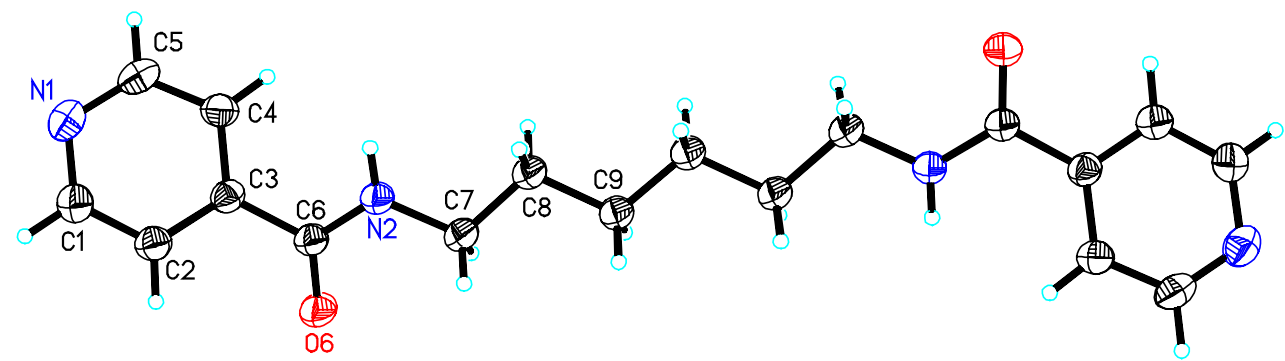

ORTEP drawing for 2c

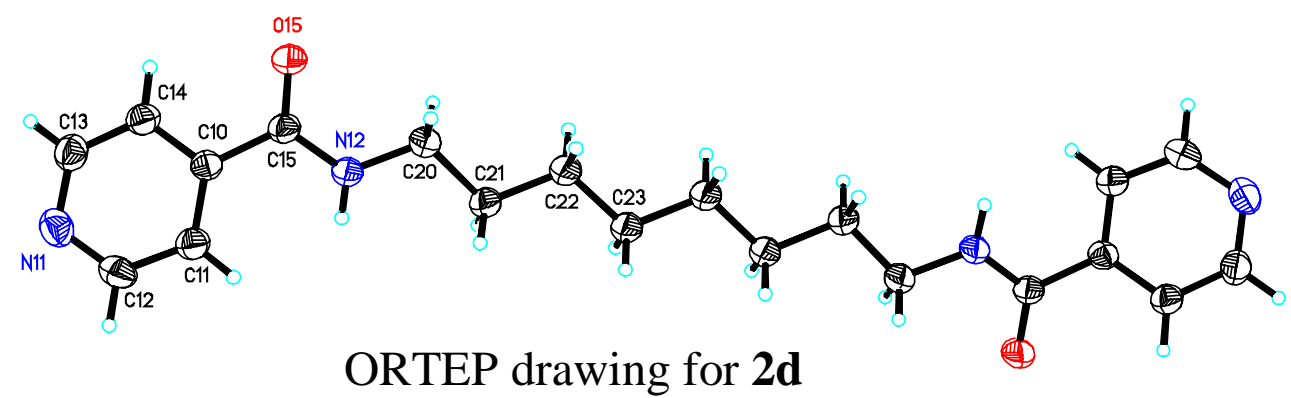




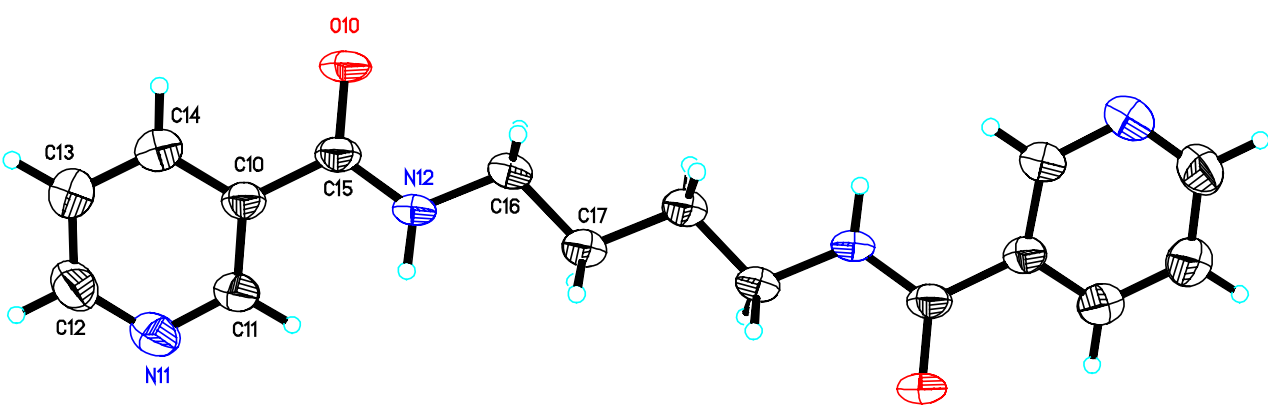

ORTEP drawing for $\mathbf{3 b}$

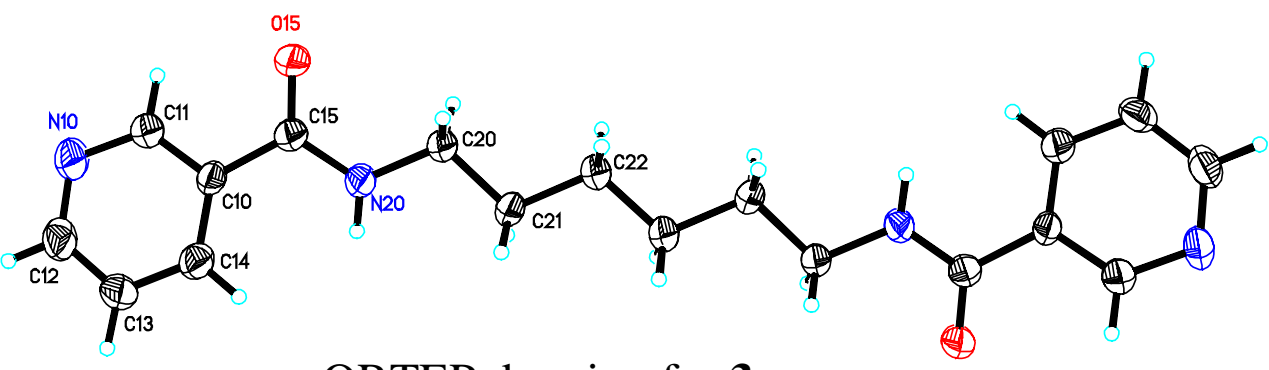

ORTEP drawing for $\mathbf{3 c}$

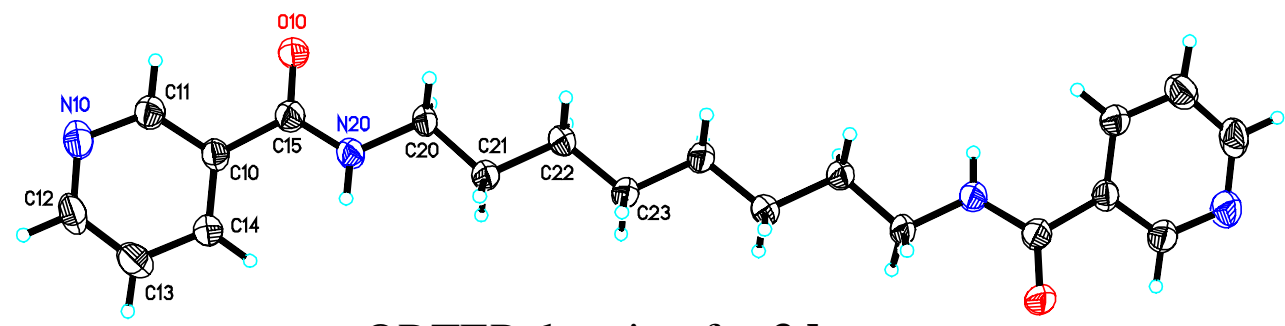

ORTEP drawing for $\mathbf{3 d}$

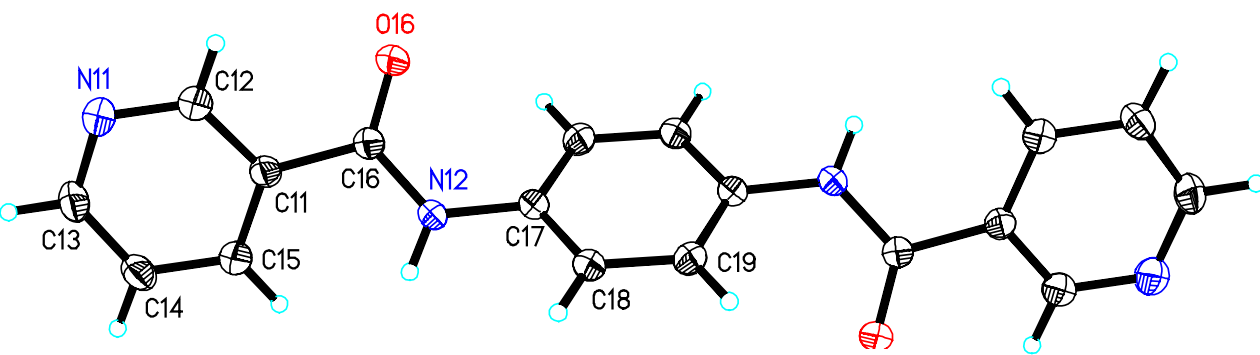

ORTEP drawing for $\mathbf{3 e}$ 
Table 1. Crystal data and structure refinement for $\mathbf{2 a}$.

Identification code

2a

Empirical formula

C14 H14 N4 O2

Formula weight

270.29

Temperature

293(2) K

Wavelength

$0.71073 \AA$

Crystal system

Monoclinic

Space group

Unit cell dimensions

$\mathrm{P} 2(1) / \mathrm{n}$

$$
\begin{array}{ll}
\mathrm{a}=4.2660(9) \AA & \alpha=90^{\circ} . \\
\mathrm{b}=15.070(3) \AA & \beta=100.73(3)^{\circ} . \\
\mathrm{c}=10.262(2) \AA & \gamma=90^{\circ} .
\end{array}
$$

Volume

$648.2(2) \AA^{3}$

$\mathrm{Z}$

2

Density (calculated)

$1.385 \mathrm{Mg} / \mathrm{m}^{3}$

Absorption coefficient

$0.097 \mathrm{~mm}^{-1}$

$\mathrm{F}(000)$

284

Crystal size

$0.15 \times 0.10 \times 0.10 \mathrm{~mm}^{3}$

Theta range for data collection

2.43 to $24.98^{\circ}$.

Index ranges

$-5<=\mathrm{h}<=0,0<=\mathrm{k}<=17,-11<=\mathrm{l}<=12$

Reflections collected

1286

Independent reflections

$1129[\mathrm{R}($ int $)=0.0181]$

Completeness to theta $=24.98^{\circ}$

$99.9 \%$

Absorption correction

None

Refinement method

Full-matrix least-squares on $\mathrm{F}^{2}$

Data / restraints / parameters

$1129 / 0 / 95$

Goodness-of-fit on $\mathrm{F}^{2}$

1.172

Final $\mathrm{R}$ indices [I $>2 \operatorname{sigma(I)]~}$

$\mathrm{R} 1=0.0423, \mathrm{wR} 2=0.0878$

$\mathrm{R}$ indices (all data)

$\mathrm{R} 1=0.0828, w \mathrm{R} 2=0.1169$

Extinction coefficient

0.011(3)

Largest diff. peak and hole

0.204 and -0.187 e. $\AA^{-3}$ 
Table 2. Atomic coordinates ( $\mathrm{x} 10^{4}$ ) and equivalent isotropic displacement parameters $\left(\AA^{2} \times 10^{3}\right)$ for $2 \mathbf{a}$. $U(\mathrm{eq})$ is defined as one third of the trace of the orthogonalized $U^{\mathrm{ij}}$ tensor.

\begin{tabular}{lrrrr}
\hline & $\mathrm{x}$ & $\mathrm{y}$ & $\mathrm{z}$ & $\mathrm{U}(\mathrm{eq})$ \\
\hline $\mathrm{O}(1)$ & $1163(5)$ & $9774(1)$ & $2261(2)$ & $56(1)$ \\
$\mathrm{N}(1)$ & $4879(6)$ & $12464(2)$ & $135(2)$ & $56(1)$ \\
$\mathrm{N}(2)$ & $247(5)$ & $10839(1)$ & $3679(2)$ & $39(1)$ \\
$\mathrm{C}(1)$ & $4215(7)$ & $11632(2)$ & $-237(2)$ & $54(1)$ \\
$\mathrm{C}(2)$ & $3083(7)$ & $11010(2)$ & $545(2)$ & $47(1)$ \\
$\mathrm{C}(3)$ & $2564(5)$ & $11247(2)$ & $1789(2)$ & $35(1)$ \\
$\mathrm{C}(4)$ & $3293(7)$ & $12106(2)$ & $2197(2)$ & $47(1)$ \\
$\mathrm{C}(5)$ & $4419(7)$ & $12685(2)$ & $1346(3)$ & $56(1)$ \\
$\mathrm{C}(6)$ & $1271(6)$ & $10554(2)$ & $2596(2)$ & $37(1)$ \\
$\mathrm{C}(7)$ & $-1218(6)$ & $10230(2)$ & $4486(2)$ & $40(1)$ \\
& & & & \\
\hline
\end{tabular}

Table 3. Bond lengths $[\AA ̊]$ and angles $\left[^{\circ}\right]$ for $\mathbf{2 a}$.

\begin{tabular}{llll}
\hline $\mathrm{O}(1)-\mathrm{C}(6)$ & $1.223(3)$ & $\mathrm{C}(6)-\mathrm{N}(2)-\mathrm{C}(7)$ & $120.8(2)$ \\
$\mathrm{N}(1)-\mathrm{C}(1)$ & $1.326(3)$ & $\mathrm{N}(1)-\mathrm{C}(1)-\mathrm{C}(2)$ & $123.7(2)$ \\
$\mathrm{N}(1)-\mathrm{C}(5)$ & $1.336(3)$ & $\mathrm{C}(1)-\mathrm{C}(2)-\mathrm{C}(3)$ & $119.7(2)$ \\
$\mathrm{N}(2)-\mathrm{C}(6)$ & $1.339(3)$ & $\mathrm{C}(4)-\mathrm{C}(3)-\mathrm{C}(2)$ & $117.2(2)$ \\
$\mathrm{N}(2)-\mathrm{C}(7)$ & $1.452(3)$ & $\mathrm{C}(4)-\mathrm{C}(3)-\mathrm{C}(6)$ & $124.8(2)$ \\
$\mathrm{C}(1)-\mathrm{C}(2)$ & $1.379(3)$ & $\mathrm{C}(2)-\mathrm{C}(3)-\mathrm{C}(6)$ & $118.0(2)$ \\
$\mathrm{C}(2)-\mathrm{C}(3)$ & $1.383(3)$ & $\mathrm{C}(3)-\mathrm{C}(4)-\mathrm{C}(5)$ & $119.1(2)$ \\
$\mathrm{C}(3)-\mathrm{C}(4)$ & $1.379(3)$ & $\mathrm{N}(1)-\mathrm{C}(5)-\mathrm{C}(4)$ & $124.0(2)$ \\
$\mathrm{C}(3)-\mathrm{C}(6)$ & $1.500(3)$ & $\mathrm{O}(1)-\mathrm{C}(6)-\mathrm{N}(2)$ & $122.7(2)$ \\
$\mathrm{C}(4)-\mathrm{C}(5)$ & $1.382(3)$ & $\mathrm{O}(1)-\mathrm{C}(6)-\mathrm{C}(3)$ & $120.8(2)$ \\
$\mathrm{C}(7)-\mathrm{C}(7) \# 1$ & $1.506(5)$ & $\mathrm{N}(2)-\mathrm{C}(6)-\mathrm{C}(3)$ & $116.5(2)$ \\
& & $\mathrm{N}(2)-\mathrm{C}(7)-\mathrm{C}(7) \# 1$ & $112.1(3)$ \\
$\mathrm{C}(1)-\mathrm{N}(1)-\mathrm{C}(5)$ & &
\end{tabular}

Symmetry transformations used to generate equivalent atoms:

$\# 1-x,-y+2,-z+1$ 
Table 4. Anisotropic displacement parameters $\left(\AA^{2} \times 10^{3}\right)$ for 2a. The anisotropic displacement factor exponent takes the form: $-2 \pi^{2}\left[h^{2} a^{* 2} U^{11}+\ldots+2 h k a^{*} b^{*} U^{12}\right]$

\begin{tabular}{lllllll}
\hline & $\mathrm{U}^{11}$ & $\mathrm{U}^{22}$ & $\mathrm{U}^{33}$ & $\mathrm{U}^{23}$ & $\mathrm{U}^{13}$ & $\mathrm{U}^{12}$ \\
\hline $\mathrm{O}(1)$ & $88(2)$ & $38(1)$ & $48(1)$ & $-6(1)$ & $27(1)$ & $-10(1)$ \\
$\mathrm{N}(1)$ & $78(2)$ & $51(1)$ & $43(1)$ & $6(1)$ & $24(1)$ & $-6(1)$ \\
$\mathrm{N}(2)$ & $50(1)$ & $35(1)$ & $35(1)$ & $4(1)$ & $17(1)$ & $1(1)$ \\
$\mathrm{C}(1)$ & $81(2)$ & $53(2)$ & $34(1)$ & $-1(1)$ & $23(1)$ & $-7(2)$ \\
$\mathrm{C}(2)$ & $66(2)$ & $42(1)$ & $36(1)$ & $-3(1)$ & $17(1)$ & $-5(1)$ \\
$\mathrm{C}(3)$ & $40(1)$ & $38(1)$ & $30(1)$ & $1(1)$ & $9(1)$ & $3(1)$ \\
$\mathrm{C}(4)$ & $69(2)$ & $43(1)$ & $32(1)$ & $-2(1)$ & $19(1)$ & $-3(1)$ \\
$\mathrm{C}(5)$ & $83(2)$ & $42(2)$ & $48(2)$ & $0(1)$ & $24(2)$ & $-9(1)$ \\
$\mathrm{C}(6)$ & $41(1)$ & $39(1)$ & $32(1)$ & $1(1)$ & $8(1)$ & $-2(1)$ \\
$\mathrm{C}(7)$ & $41(1)$ & $45(1)$ & $37(1)$ & $4(1)$ & $15(1)$ & $-2(1)$ \\
& & & & & & \\
\hline
\end{tabular}

Table 5. Hydrogen coordinates ( x 104) and isotropic displacement parameters $\left(\AA^{2} \times 10^{3}\right)$ for $\mathbf{2 a}$.

\begin{tabular}{lcccc}
\hline & $x$ & $y$ & $z$ & $U(e q)$ \\
& & & & \\
$H(2 N)$ & $260(60)$ & $11420(18)$ & $3880(20)$ & 50 \\
$H(1)$ & 4531 & 11458 & -1072 & 65 \\
$H(2)$ & 2670 & 10433 & 237 & 57 \\
$H(4)$ & 3031 & 12294 & 3034 & 56 \\
$H(5)$ & 4884 & 13263 & 1634 & 67 \\
$H(7 A)$ & -2437 & 9789 & 3916 & 48 \\
$H(7 B)$ & -2686 & 10556 & 4925 & 48 \\
& & & & \\
\hline
\end{tabular}


Table 1. Crystal data and structure refinement for $\mathbf{2 b}$.

Identification code

Empirical formula

Formula weight

Temperature

Wavelength

Crystal system

Space group

Unit cell dimensions

Volume

$\mathrm{Z}$

Density (calculated)

Absorption coefficient

$\mathrm{F}(000)$

Crystal size

Theta range for data collection

Index ranges

Reflections collected

Independent reflections

Completeness to theta $=24.99^{\circ}$

Absorption correction

Refinement method

Data / restraints / parameters

Goodness-of-fit on $\mathrm{F}^{2}$

Final $\mathrm{R}$ indices [I $>2 \operatorname{sigma}(\mathrm{I})]$

$\mathrm{R}$ indices (all data)

Extinction coefficient

Largest diff. peak and hole 2b

C16 H18 N4 O2

298.34

293(2) K

$0.71073 \AA$

Monoclinic

$\mathrm{C} 2 / \mathrm{c}$

$\mathrm{a}=26.519(5) \AA$

$\alpha=90^{\circ}$.

$\mathrm{b}=5.6370(11) \AA$

$\beta=101.36(3)^{\circ}$.

$\mathrm{c}=10.084(2) \AA$

1477.9(5) $\AA^{3}$

4

$1.341 \mathrm{Mg} / \mathrm{m}^{3}$

$0.092 \mathrm{~mm}^{-1}$

632

$0.15 \times 0.10 \times 0.10 \mathrm{~mm}^{3}$

1.57 to $24.99^{\circ}$.

$0<=\mathrm{h}<=31,0<=\mathrm{k}<=6,-11<=\mathrm{l}<=11$

1324

$1294[\mathrm{R}($ int $)=0.0108]$

$99.9 \%$

None

Full-matrix least-squares on $\mathrm{F}^{2}$

1294 / 0 / 101

0.994

$\mathrm{R} 1=0.0459, \mathrm{wR} 2=0.1107$

$\mathrm{R} 1=0.0769, \mathrm{wR} 2=0.1501$

$0.012(2)$

0.209 and $-0.189 \mathrm{e} . \AA^{-3}$ 
Table 2. Atomic coordinates ( $\left.\mathrm{x} 10^{4}\right)$ and equivalent isotropic displacement parameters $\left(\AA^{2} \times 10^{3}\right)$ for $\mathbf{2 b}$. $U(e q)$ is defined as one third of the trace of the orthogonalized $U^{\mathrm{ij}}$ tensor.

\begin{tabular}{lcrrr}
\hline & $\mathrm{x}$ & $\mathrm{y}$ & $\mathrm{z}$ & $\mathrm{U}(\mathrm{eq})$ \\
\hline $\mathrm{N}(11)$ & $2788(1)$ & $-5039(4)$ & $4207(2)$ & $52(1)$ \\
$\mathrm{N}(12)$ & $4235(1)$ & $916(4)$ & $5210(2)$ & $39(1)$ \\
$\mathrm{O}(15)$ & $4143(1)$ & $-324(4)$ & $7270(2)$ & $53(1)$ \\
$\mathrm{C}(10)$ & $3580(1)$ & $-1980(4)$ & $5359(2)$ & $34(1)$ \\
$\mathrm{C}(11)$ & $3266(1)$ & $-1463(5)$ & $4130(2)$ & $40(1)$ \\
$\mathrm{C}(12)$ & $2874(1)$ & $-3005(5)$ & $3608(3)$ & $45(1)$ \\
$\mathrm{C}(13)$ & $3096(1)$ & $-5510(5)$ & $5393(3)$ & $50(1)$ \\
$\mathrm{C}(14)$ & $3486(1)$ & $-4055(4)$ & $6012(3)$ & $42(1)$ \\
$\mathrm{C}(15)$ & $4009(1)$ & $-392(4)$ & $6026(2)$ & $37(1)$ \\
$\mathrm{C}(16)$ & $4654(1)$ & $2518(4)$ & $5763(2)$ & $39(1)$ \\
$\mathrm{C}(17)$ & $4782(1)$ & $4171(4)$ & $4700(2)$ & $41(1)$ \\
& & & & \\
\hline
\end{tabular}

Table 3. Bond lengths $[\AA]$ and angles $\left[^{\circ}\right]$ for $\mathbf{2 b}$.

\begin{tabular}{llll}
\hline $\mathrm{N}(11)-\mathrm{C}(12)$ & $1.336(4)$ & $\mathrm{C}(15)-\mathrm{N}(12)-\mathrm{C}(16)$ & $120.67(19)$ \\
$\mathrm{N}(11)-\mathrm{C}(13)$ & $1.335(4)$ & $\mathrm{C}(11)-\mathrm{C}(10)-\mathrm{C}(14)$ & $118.1(2)$ \\
$\mathrm{N}(12)-\mathrm{C}(15)$ & $1.332(3)$ & $\mathrm{C}(11)-\mathrm{C}(10)-\mathrm{C}(15)$ & $123.1(2)$ \\
$\mathrm{N}(12)-\mathrm{C}(16)$ & $1.455(3)$ & $\mathrm{C}(14)-\mathrm{C}(10)-\mathrm{C}(15)$ & $118.8(2)$ \\
$\mathrm{O}(15)-\mathrm{C}(15)$ & $1.236(3)$ & $\mathrm{C}(10)-\mathrm{C}(11)-\mathrm{C}(12)$ & $119.0(2)$ \\
$\mathrm{C}(10)-\mathrm{C}(11)$ & $1.381(3)$ & $\mathrm{N}(11)-\mathrm{C}(12)-\mathrm{C}(11)$ & $123.6(2)$ \\
$\mathrm{C}(10)-\mathrm{C}(14)$ & $1.389(3)$ & $\mathrm{N}(11)-\mathrm{C}(13)-\mathrm{C}(14)$ & $124.2(2)$ \\
$\mathrm{C}(10)-\mathrm{C}(15)$ & $1.498(3)$ & $\mathrm{C}(13)-\mathrm{C}(14)-\mathrm{C}(10)$ & $118.5(2)$ \\
$\mathrm{C}(11)-\mathrm{C}(12)$ & $1.377(3)$ & $\mathrm{O}(15)-\mathrm{C}(15)-\mathrm{N}(12)$ & $122.4(2)$ \\
$\mathrm{C}(13)-\mathrm{C}(14)$ & $1.370(4)$ & $\mathrm{O}(15)-\mathrm{C}(15)-\mathrm{C}(10)$ & $121.0(2)$ \\
$\mathrm{C}(16)-\mathrm{C}(17)$ & $1.509(3)$ & $\mathrm{N}(12)-\mathrm{C}(15)-\mathrm{C}(10)$ & $116.6(2)$ \\
$\mathrm{C}(17)-\mathrm{C}(17) \# 1$ & $1.517(5)$ & $\mathrm{N}(12)-\mathrm{C}(16)-\mathrm{C}(17)$ & $111.93(19)$ \\
& & $\mathrm{C}(16)-\mathrm{C}(17)-\mathrm{C}(17) \# 1$ & $111.0(2)$ \\
$\mathrm{C}(12)-\mathrm{N}(11)-\mathrm{C}(13)$ & $116.5(2)$ & &
\end{tabular}


Table 4. Anisotropic displacement parameters $\left(\AA^{2} \times 10^{3}\right)$ for $\mathbf{2 b}$. The anisotropic displacement factor exponent takes the form: $-2 \pi^{2}\left[h^{2} a^{* 2} U^{11}+\ldots+2 h k a^{*} b^{*} U^{12}\right]$

\begin{tabular}{lllllll}
\hline & $\mathrm{U}^{11}$ & $\mathrm{U}^{22}$ & $\mathrm{U}^{33}$ & $\mathrm{U}^{23}$ & $\mathrm{U}^{13}$ & $\mathrm{U}^{12}$ \\
\hline $\mathrm{N}(11)$ & $56(1)$ & $52(1)$ & $49(1)$ & $-7(1)$ & $14(1)$ & $-16(1)$ \\
$\mathrm{N}(12)$ & $46(1)$ & $44(1)$ & $27(1)$ & $0(1)$ & $7(1)$ & $-10(1)$ \\
$\mathrm{O}(15)$ & $60(1)$ & $70(1)$ & $27(1)$ & $5(1)$ & $5(1)$ & $-17(1)$ \\
$\mathrm{C}(10)$ & $36(1)$ & $36(1)$ & $32(1)$ & $-2(1)$ & $9(1)$ & $-1(1)$ \\
$\mathrm{C}(11)$ & $46(1)$ & $39(1)$ & $36(1)$ & $2(1)$ & $8(1)$ & $-6(1)$ \\
$\mathrm{C}(12)$ & $46(1)$ & $52(2)$ & $36(1)$ & $-2(1)$ & $4(1)$ & $-4(1)$ \\
$\mathrm{C}(13)$ & $61(2)$ & $41(1)$ & $51(2)$ & $1(1)$ & $15(1)$ & $-12(1)$ \\
$\mathrm{C}(14)$ & $47(1)$ & $42(1)$ & $37(1)$ & $6(1)$ & $9(1)$ & $-6(1)$ \\
$\mathrm{C}(15)$ & $40(1)$ & $40(1)$ & $30(1)$ & $0(1)$ & $7(1)$ & $-3(1)$ \\
$\mathrm{C}(16)$ & $42(1)$ & $42(1)$ & $32(1)$ & $-2(1)$ & $6(1)$ & $-11(1)$ \\
$\mathrm{C}(17)$ & $47(1)$ & $41(1)$ & $34(1)$ & $0(1)$ & $7(1)$ & $-9(1)$ \\
& & & & & & \\
\hline
\end{tabular}

Table 5. Hydrogen coordinates ( $\left.\mathrm{x} 10^{4}\right)$ and isotropic displacement parameters $\left(\AA^{2} \times 10^{3}\right)$ for $\mathbf{2 b}$.

\begin{tabular}{lrrrr}
\hline & \multicolumn{1}{c}{$\mathrm{x}$} & $\mathrm{z}$ & $\mathrm{U}(\mathrm{eq})$ \\
& & & & \\
$\mathrm{H}(12)$ & 4132 & 812 & 4348 & 47 \\
$\mathrm{H}(11)$ & 3319 & -94 & 3660 & 48 \\
$\mathrm{H}(12 \mathrm{~A})$ & 2658 & -2610 & 2796 & 54 \\
$\mathrm{H}(13)$ & 3042 & -6916 & 5827 & 60 \\
$\mathrm{H}(14)$ & 3684 & -4449 & 6851 & 50 \\
$\mathrm{H}(16 \mathrm{~A})$ & 4956 & 1596 & 6146 & 46 \\
$\mathrm{H}(16 \mathrm{~B})$ & 4558 & 3445 & 6485 & 46 \\
$\mathrm{H}(17 \mathrm{~A})$ & 4480 & 5096 & 4315 & 49 \\
$\mathrm{H}(17 \mathrm{~B})$ & 4880 & 3249 & 3979 & 49 \\
& & & & \\
\hline
\end{tabular}


Table 1. Crystal data and structure refinement for $\mathbf{2 c}$.

Identification code

Empirical formula

Formula weight

Temperature

Wavelength

Crystal system

Space group

Unit cell dimensions

Volume

Z

Density (calculated)

Absorption coefficient

$\mathrm{F}(000)$

Crystal size

Theta range for data collection

Index ranges

Reflections collected

Independent reflections

Completeness to theta $=24.99^{\circ}$

Absorption correction

Refinement method

Data / restraints / parameters

Goodness-of-fit on $\mathrm{F}^{2}$

Final $\mathrm{R}$ indices [I $>2 \operatorname{sigma}(\mathrm{I})]$

$\mathrm{R}$ indices (all data)

Extinction coefficient

Largest diff. peak and hole
$2 c$

C18 H22 N4 O2

326.40

293(2) K

$0.71073 \AA$

Monoclinic

$\mathrm{P} 2(1) / \mathrm{c}$

$\mathrm{a}=5.1020(10) \AA$

$\alpha=90^{\circ}$.

$\mathrm{b}=5.2790(11) \AA$

$\beta=91.04(3)^{\circ}$.

$\mathrm{c}=30.784(6) \AA$

$829.0(3) \AA^{3}$

2

$1.308 \mathrm{Mg} / \mathrm{m}^{3}$

$0.088 \mathrm{~mm}^{-1}$

348

$0.15 \times 0.10 \times 0.10 \mathrm{~mm}^{3}$

1.32 to $24.99^{\circ}$.

$0<=\mathrm{h}<=6,0<=\mathrm{k}<=6,-36<=1<=36$

1632

$1453[\mathrm{R}($ int $)=0.0228]$

$99.9 \%$

None

Full-matrix least-squares on $\mathrm{F}^{2}$

$1453 / 0 / 110$

1.143

$\mathrm{R} 1=0.0408, \mathrm{wR} 2=0.0820$

$\mathrm{R} 1=0.0798, \mathrm{wR} 2=0.1170$

$0.027(3)$

0.183 and $-0.192 \mathrm{e} . \AA^{-3}$ 
Table 2. Atomic coordinates ( $\mathrm{x} 10^{4}$ ) and equivalent isotropic displacement parameters $\left(\AA^{2} \times 10^{3}\right.$ ) for 2 c. $U(e q)$ is defined as one third of the trace of the orthogonalized $U^{i j}$ tensor.

\begin{tabular}{lrrrr}
\hline & $\mathrm{x}$ & $\mathrm{y}$ & $\mathrm{z}$ & $\mathrm{U}(\mathrm{eq})$ \\
\hline $\mathrm{N}(1)$ & $-2029(4)$ & $6857(4)$ & $2209(1)$ & $54(1)$ \\
$\mathrm{N}(2)$ & $-2330(3)$ & $679(4)$ & $945(1)$ & $43(1)$ \\
$\mathrm{O}(6)$ & $1937(3)$ & $1677(4)$ & $1025(1)$ & $57(1)$ \\
$\mathrm{C}(1)$ & $-34(5)$ & $7255(5)$ & $1943(1)$ & $50(1)$ \\
$\mathrm{C}(2)$ & $539(5)$ & $5714(5)$ & $1596(1)$ & $45(1)$ \\
$\mathrm{C}(3)$ & $-1031(4)$ & $3635(5)$ & $1509(1)$ & $38(1)$ \\
$\mathrm{C}(4)$ & $-3131(4)$ & $3202(5)$ & $1781(1)$ & $45(1)$ \\
$\mathrm{C}(5)$ & $-3520(5)$ & $4858(5)$ & $2123(1)$ & $51(1)$ \\
$\mathrm{C}(6)$ & $-365(4)$ & $1925(5)$ & $1139(1)$ & $41(1)$ \\
$\mathrm{C}(7)$ & $-1847(4)$ & $-1092(5)$ & $592(1)$ & $46(1)$ \\
$\mathrm{C}(8)$ & $-4287(4)$ & $-2538(5)$ & $459(1)$ & $44(1)$ \\
$\mathrm{C}(9)$ & $-3783(4)$ & $-4294(5)$ & $77(1)$ & $43(1)$ \\
& & & & \\
\hline
\end{tabular}

Table 3. Bond lengths $[\AA]$ and angles $\left[{ }^{\circ}\right]$ for $\mathbf{2 c}$.

\begin{tabular}{llll}
\hline $\mathrm{N}(1)-\mathrm{C}(5)$ & $1.325(3)$ & $\mathrm{C}(5)-\mathrm{N}(1)-\mathrm{C}(1)$ & $116.5(2)$ \\
$\mathrm{N}(1)-\mathrm{C}(1)$ & $1.333(3)$ & $\mathrm{C}(6)-\mathrm{N}(2)-\mathrm{C}(7)$ & $121.01(19)$ \\
$\mathrm{N}(2)-\mathrm{C}(6)$ & $1.331(3)$ & $\mathrm{N}(1)-\mathrm{C}(1)-\mathrm{C}(2)$ & $123.9(2)$ \\
$\mathrm{N}(2)-\mathrm{C}(7)$ & $1.457(3)$ & $\mathrm{C}(1)-\mathrm{C}(2)-\mathrm{C}(3)$ & $119.2(2)$ \\
$\mathrm{O}(6)-\mathrm{C}(6)$ & $1.239(3)$ & $\mathrm{C}(2)-\mathrm{C}(3)-\mathrm{C}(4)$ & $117.6(2)$ \\
$\mathrm{C}(1)-\mathrm{C}(2)$ & $1.380(3)$ & $\mathrm{C}(2)-\mathrm{C}(3)-\mathrm{C}(6)$ & $119.2(2)$ \\
$\mathrm{C}(2)-\mathrm{C}(3)$ & $1.382(3)$ & $\mathrm{C}(4)-\mathrm{C}(3)-\mathrm{C}(6)$ & $123.2(2)$ \\
$\mathrm{C}(3)-\mathrm{C}(4)$ & $1.392(3)$ & $\mathrm{C}(5)-\mathrm{C}(4)-\mathrm{C}(3)$ & $118.5(2)$ \\
$\mathrm{C}(3)-\mathrm{C}(6)$ & $1.497(3)$ & $\mathrm{N}(1)-\mathrm{C}(5)-\mathrm{C}(4)$ & $124.4(2)$ \\
$\mathrm{C}(4)-\mathrm{C}(5)$ & $1.385(3)$ & $\mathrm{O}(6)-\mathrm{C}(6)-\mathrm{N}(2)$ & $122.1(2)$ \\
$\mathrm{C}(7)-\mathrm{C}(8)$ & $1.511(3)$ & $\mathrm{O}(6)-\mathrm{C}(6)-\mathrm{C}(3)$ & $120.6(2)$ \\
$\mathrm{C}(8)-\mathrm{C}(9)$ & $1.522(3)$ & $\mathrm{N}(2)-\mathrm{C}(6)-\mathrm{C}(3)$ & $117.34(19)$ \\
$\mathrm{C}(9)-\mathrm{C}(9) \# 1$ & $1.516(4)$ & $\mathrm{N}(2)-\mathrm{C}(7)-\mathrm{C}(8)$ & $112.17(18)$
\end{tabular}




$\begin{array}{ll}\mathrm{C}(7)-\mathrm{C}(8)-\mathrm{C}(9) & 111.58(18) \\ \mathrm{C}(9) \# 1-\mathrm{C}(9)-\mathrm{C}(8) & 113.0(2)\end{array}$

Symmetry transformations used to generate equivalent atoms:

$\# 1-\mathrm{x}-1,-\mathrm{y}-1,-\mathrm{z}$

Table 4. Anisotropic displacement parameters $\left(\AA^{2} \times 10^{3}\right)$ for $2 \mathrm{c}$. The anisotropic displacement factor exponent takes the form: $-2 \pi^{2}\left[h^{2} a^{* 2} U^{11}+\ldots+2 h k a^{*} b^{*} U^{12}\right]$

\begin{tabular}{lllllll}
\hline & $\mathrm{U}^{11}$ & $\mathrm{U}^{22}$ & $\mathrm{U}^{33}$ & $\mathrm{U}^{23}$ & $\mathrm{U}^{13}$ & $\mathrm{U}^{12}$ \\
\hline $\mathrm{N}(1)$ & $59(1)$ & $55(1)$ & $47(1)$ & $-10(1)$ & $5(1)$ & $6(1)$ \\
$\mathrm{N}(2)$ & $31(1)$ & $51(1)$ & $46(1)$ & $-12(1)$ & $4(1)$ & $-1(1)$ \\
$\mathrm{O}(6)$ & $32(1)$ & $76(1)$ & $64(1)$ & $-25(1)$ & $9(1)$ & $-2(1)$ \\
$\mathrm{C}(1)$ & $51(1)$ & $50(2)$ & $49(1)$ & $-6(1)$ & $1(1)$ & $-3(1)$ \\
$\mathrm{C}(2)$ & $40(1)$ & $49(2)$ & $44(1)$ & $-2(1)$ & $4(1)$ & $0(1)$ \\
$\mathrm{C}(3)$ & $35(1)$ & $44(1)$ & $36(1)$ & $-3(1)$ & $1(1)$ & $3(1)$ \\
$\mathrm{C}(4)$ & $40(1)$ & $52(2)$ & $44(1)$ & $1(1)$ & $5(1)$ & $-4(1)$ \\
$\mathrm{C}(5)$ & $50(1)$ & $62(2)$ & $41(1)$ & $0(1)$ & $11(1)$ & $9(1)$ \\
$\mathrm{C}(6)$ & $34(1)$ & $48(2)$ & $41(1)$ & $-6(1)$ & $4(1)$ & $-1(1)$ \\
$\mathrm{C}(7)$ & $39(1)$ & $56(2)$ & $45(1)$ & $-13(1)$ & $8(1)$ & $-1(1)$ \\
$\mathrm{C}(8)$ & $37(1)$ & $50(2)$ & $44(1)$ & $-10(1)$ & $5(1)$ & $0(1)$ \\
$\mathrm{C}(9)$ & $36(1)$ & $46(1)$ & $47(1)$ & $-9(1)$ & $4(1)$ & $2(1)$ \\
& & & & & & \\
\hline
\end{tabular}


Table 5. Hydrogen coordinates ( x 10 $0^{4}$ ) and isotropic displacement parameters $\left(\AA^{2} \times 10^{3}\right)$ for $2 c$.

\begin{tabular}{lrrrr}
\hline & $\mathrm{x}$ & $\mathrm{y}$ & $\mathrm{z}$ & $\mathrm{U}(\mathrm{eq})$ \\
& & & & \\
$\mathrm{H}(2)$ & -3908 & 923 & 1029 & 51 \\
$\mathrm{H}(1)$ & 1035 & 8654 & 1996 & 60 \\
$\mathrm{H}(2 \mathrm{~A})$ & 1965 & 6071 & 1422 & 53 \\
$\mathrm{H}(4)$ & -4247 & 1831 & 1735 & 54 \\
$\mathrm{H}(5)$ & -4922 & 4546 & 2304 & 61 \\
$\mathrm{H}(7 \mathrm{~A})$ & -498 & -2283 & 683 & 56 \\
$\mathrm{H}(7 \mathrm{~B})$ & -1205 & -167 & 343 & 56 \\
$\mathrm{H}(8 \mathrm{~A})$ & -5663 & -1349 & 379 & 52 \\
$\mathrm{H}(8 \mathrm{~B})$ & -4886 & -3528 & 703 & 52 \\
$\mathrm{H}(9 \mathrm{~A})$ & -3114 & -3305 & -162 & 52 \\
$\mathrm{H}(9 \mathrm{~B})$ & -2447 & -5514 & 161 & 52 \\
& & & & \\
\hline
\end{tabular}


Table 1. Crystal data and structure refinement for $\mathbf{2 d}$.

Identification code

Empirical formula

Formula weight

Temperature

Wavelength

Crystal system

Space group

Unit cell dimensions

Volume

$\mathrm{Z}$

Density (calculated)

Absorption coefficient

$\mathrm{F}(000)$

Crystal size

Theta range for data collection

Index ranges

Reflections collected

Independent reflections

Completeness to theta $=25.00^{\circ}$

Absorption correction

Refinement method

Data / restraints / parameters

Goodness-of-fit on $\mathrm{F}^{2}$

Final $\mathrm{R}$ indices [I $>2 \operatorname{sigma}(\mathrm{I})]$

$\mathrm{R}$ indices (all data)

Largest diff. peak and hole 2d

C20 H26 N4 O2

354.45

293(2) K

$0.71073 \AA$

Monoclinic

$\mathrm{P} 2(1) / \mathrm{c}$

$a=5.1130(10) \AA \quad \alpha=90^{\circ}$.

$\mathrm{b}=5.3230(11) \AA$

$\beta=92.36(3)^{\circ}$.

$\mathrm{c}=33.792(7) \AA$

$\gamma=90^{\circ}$.

918.9(3) $\AA^{3}$

2

$1.281 \mathrm{Mg} / \mathrm{m}^{3}$

$0.085 \mathrm{~mm}^{-1}$

380

$0.20 \times 0.10 \times 0.10 \mathrm{~mm}^{3}$

1.21 to $25.00^{\circ}$.

$0<=\mathrm{h}<=6,-6<=\mathrm{k}<=6,-40<=1<=40$

3392

$1608[\mathrm{R}($ int $)=0.0306]$

$99.7 \%$

None

Full-matrix least-squares on $\mathrm{F}^{2}$

$1608 / 0 / 118$

1.083

$\mathrm{R} 1=0.0408, \mathrm{wR} 2=0.1043$

$\mathrm{R} 1=0.0611, \mathrm{wR} 2=0.1291$

0.159 and -0.169 e. $\AA^{-3}$ 
Table 2. Atomic coordinates ( $\mathrm{x} 10^{4}$ ) and equivalent isotropic displacement parameters $\left(\AA^{2} \times 10^{3}\right.$ ) for $\mathbf{2 d}$. $U(e q)$ is defined as one third of the trace of the orthogonalized $U^{i j}$ tensor.

\begin{tabular}{lcccc}
\hline & $\mathrm{x}$ & $\mathrm{y}$ & $\mathrm{z}$ & $\mathrm{U}(\mathrm{eq})$ \\
\hline $\mathrm{N}(11)$ & $3065(3)$ & $-3428(3)$ & $2763(1)$ & $54(1)$ \\
$\mathrm{N}(12)$ & $3209(3)$ & $2776(3)$ & $3901(1)$ & $43(1)$ \\
$\mathrm{C}(10)$ & $4308(3)$ & $-186(3)$ & $3393(1)$ & $37(1)$ \\
$\mathrm{C}(11)$ & $2126(4)$ & $227(4)$ & $3146(1)$ & $45(1)$ \\
$\mathrm{C}(12)$ & $1596(4)$ & $-1413(4)$ & $2839(1)$ & $50(1)$ \\
$\mathrm{C}(13)$ & $5156(4)$ & $-3807(4)$ & $3004(1)$ & $50(1)$ \\
$\mathrm{C}(14)$ & $5855(4)$ & $-2257(4)$ & $3315(1)$ & $44(1)$ \\
$\mathrm{C}(15)$ & $5108(3)$ & $1527(3)$ & $3725(1)$ & $40(1)$ \\
$\mathrm{O}(15)$ & $7437(2)$ & $1777(3)$ & $3829(1)$ & $58(1)$ \\
$\mathrm{C}(20)$ & $3801(3)$ & $4532(4)$ & $4219(1)$ & $45(1)$ \\
$\mathrm{C}(21)$ & $1425(3)$ & $5988(4)$ & $4337(1)$ & $44(1)$ \\
$\mathrm{C}(22)$ & $2053(3)$ & $7735(3)$ & $4682(1)$ & $44(1)$ \\
$\mathrm{C}(23)$ & $-312(3)$ & $9141(4)$ & $4826(1)$ & $45(1)$ \\
& & & & \\
\hline
\end{tabular}

Table 3. Bond lengths $[\AA]$ and angles $\left[{ }^{\circ}\right]$ for $\mathbf{2 d}$.

\begin{tabular}{llll}
\hline $\mathrm{N}(11)-\mathrm{C}(13)$ & $1.331(3)$ & & \\
$\mathrm{N}(11)-\mathrm{C}(12)$ & $1.341(3)$ & $\mathrm{C}(13)-\mathrm{N}(11)-\mathrm{C}(12)$ & $116.54(17)$ \\
$\mathrm{N}(12)-\mathrm{C}(15)$ & $1.337(2)$ & $\mathrm{C}(15)-\mathrm{N}(12)-\mathrm{C}(20)$ & $121.25(15)$ \\
$\mathrm{N}(12)-\mathrm{C}(20)$ & $1.446(2)$ & $\mathrm{C}(11)-\mathrm{C}(10)-\mathrm{C}(14)$ & $117.48(17)$ \\
$\mathrm{C}(10)-\mathrm{C}(11)$ & $1.385(3)$ & $\mathrm{C}(11)-\mathrm{C}(10)-\mathrm{C}(15)$ & $123.17(16)$ \\
$\mathrm{C}(10)-\mathrm{C}(14)$ & $1.389(3)$ & $\mathrm{C}(14)-\mathrm{C}(10)-\mathrm{C}(15)$ & $119.30(16)$ \\
$\mathrm{C}(10)-\mathrm{C}(15)$ & $1.488(2)$ & $\mathrm{C}(12)-\mathrm{C}(11)-\mathrm{C}(10)$ & $118.97(18)$ \\
$\mathrm{C}(11)-\mathrm{C}(12)$ & $1.373(3)$ & $\mathrm{N}(11)-\mathrm{C}(12)-\mathrm{C}(11)$ & $123.93(18)$ \\
$\mathrm{C}(13)-\mathrm{C}(14)$ & $1.372(3)$ & $\mathrm{N}(11)-\mathrm{C}(13)-\mathrm{C}(14)$ & $123.63(18)$ \\
$\mathrm{C}(15)-\mathrm{O}(15)$ & $1.235(2)$ & $\mathrm{C}(13)-\mathrm{C}(14)-\mathrm{C}(10)$ & $119.44(17)$ \\
$\mathrm{C}(20)-\mathrm{C}(21)$ & $1.509(3)$ & $\mathrm{O}(15)-\mathrm{C}(15)-\mathrm{N}(12)$ & $121.85(16)$ \\
$\mathrm{C}(21)-\mathrm{C}(22)$ & $1.513(3)$ & $\mathrm{O}(15)-\mathrm{C}(15)-\mathrm{C}(10)$ & $120.88(16)$ \\
$\mathrm{C}(22)-\mathrm{C}(23)$ & $1.520(3)$ & $\mathrm{N}(12)-\mathrm{C}(15)-\mathrm{C}(10)$ & $117.27(15)$ \\
$\mathrm{C}(23)-\mathrm{C}(23) \# 1$ & $1.512(4)$ & $\mathrm{N}(12)-\mathrm{C}(20)-\mathrm{C}(21)$ & $112.50(14)$
\end{tabular}




$\begin{array}{llll}\mathrm{C}(20)-\mathrm{C}(21)-\mathrm{C}(22) & 111.83(15) & \mathrm{C}(23) \# 1-\mathrm{C}(23)-\mathrm{C}(22) & 113.72(18) \\ \mathrm{C}(21)-\mathrm{C}(22)-\mathrm{C}(23) & 113.79(15) & & \end{array}$

Symmetry transformations used to generate equivalent atoms:

$\# 1-x,-y+2,-z+1$

Table 4. Anisotropic displacement parameters $\left(\AA^{2} \times 10^{3}\right)$ for $\mathbf{2 d}$. The anisotropic

displacement factor exponent takes the form: $-2 \pi^{2}\left[h^{2} a^{* 2} U^{11}+\ldots+2 h k a^{*} b^{*} U^{12}\right]$

\begin{tabular}{lllllll}
\hline & $\mathrm{U}^{11}$ & $\mathrm{U}^{22}$ & $\mathrm{U}^{33}$ & $\mathrm{U}^{23}$ & $\mathrm{U}^{13}$ & $\mathrm{U}^{12}$ \\
\hline $\mathrm{N}(11)$ & $56(1)$ & $54(1)$ & $52(1)$ & $-12(1)$ & $0(1)$ & $-8(1)$ \\
$\mathrm{N}(12)$ & $29(1)$ & $47(1)$ & $52(1)$ & $-12(1)$ & $-1(1)$ & $-2(1)$ \\
$\mathrm{C}(10)$ & $31(1)$ & $41(1)$ & $40(1)$ & $-1(1)$ & $3(1)$ & $-3(1)$ \\
$\mathrm{C}(11)$ & $39(1)$ & $46(1)$ & $50(1)$ & $-2(1)$ & $-1(1)$ & $1(1)$ \\
$\mathrm{C}(12)$ & $42(1)$ & $61(1)$ & $47(1)$ & $0(1)$ & $-6(1)$ & $-7(1)$ \\
$\mathrm{C}(13)$ & $50(1)$ & $44(1)$ & $57(1)$ & $-8(1)$ & $5(1)$ & $1(1)$ \\
$\mathrm{C}(14)$ & $38(1)$ & $44(1)$ & $50(1)$ & $-1(1)$ & $1(1)$ & $1(1)$ \\
$\mathrm{C}(15)$ & $31(1)$ & $42(1)$ & $47(1)$ & $-3(1)$ & $2(1)$ & $-1(1)$ \\
$\mathrm{O}(15)$ & $30(1)$ & $71(1)$ & $71(1)$ & $-25(1)$ & $-4(1)$ & $1(1)$ \\
$\mathrm{C}(20)$ & $36(1)$ & $49(1)$ & $50(1)$ & $-11(1)$ & $-2(1)$ & $0(1)$ \\
$\mathrm{C}(21)$ & $35(1)$ & $43(1)$ & $53(1)$ & $-7(1)$ & $0(1)$ & $2(1)$ \\
$\mathrm{C}(22)$ & $37(1)$ & $43(1)$ & $53(1)$ & $-7(1)$ & $-3(1)$ & $0(1)$ \\
$\mathrm{C}(23)$ & $36(1)$ & $44(1)$ & $55(1)$ & $-7(1)$ & $-1(1)$ & $0(1)$ \\
& & & & & & \\
\hline
\end{tabular}


Table 5. Hydrogen coordinates ( x 104) and isotropic displacement parameters $\left(\AA^{2} \times 10^{3}\right)$ for $\mathbf{2 d}$.

\begin{tabular}{|c|c|c|c|c|}
\hline & $\mathrm{x}$ & $\mathrm{y}$ & $\mathrm{z}$ & $\mathrm{U}(\mathrm{eq})$ \\
\hline $\mathrm{H}(12)$ & 1605 & 2531 & 3824 & 51 \\
\hline $\mathrm{H}(11)$ & 1037 & 1594 & 3186 & 54 \\
\hline $\mathrm{H}(12 \mathrm{~A})$ & 129 & -1107 & 2674 & 60 \\
\hline $\mathrm{H}(13)$ & 6201 & -5199 & 2958 & 60 \\
\hline $\mathrm{H}(14)$ & 7351 & -2592 & 3472 & 53 \\
\hline $\mathrm{H}(20 \mathrm{~A})$ & 5127 & 5700 & 4136 & 54 \\
\hline $\mathrm{H}(20 \mathrm{~B})$ & 4515 & 3619 & 4447 & 54 \\
\hline $\mathrm{H}(21 \mathrm{~A})$ & 70 & 4821 & 4410 & 53 \\
\hline $\mathrm{H}(21 \mathrm{~B})$ & 754 & 6962 & 4113 & 53 \\
\hline $\mathrm{H}(22 \mathrm{~A})$ & 3347 & 8948 & 4602 & 53 \\
\hline $\mathrm{H}(22 \mathrm{~B})$ & 2825 & 6764 & 4899 & 53 \\
\hline $\mathrm{H}(23 \mathrm{~A})$ & -1069 & 10131 & 4609 & 54 \\
\hline $\mathrm{H}(23 \mathrm{~B})$ & -1617 & 7929 & 4902 & 54 \\
\hline
\end{tabular}


Table 1. Crystal data and structure refinement for $\mathbf{3 b}$.

Identification code

Empirical formula

Formula weight

Temperature

Wavelength

Crystal system

Space group

Unit cell dimensions

Volume

$\mathrm{Z}$

Density (calculated)

Absorption coefficient

$\mathrm{F}(000)$

Crystal size

Theta range for data collection

Index ranges

Reflections collected

Independent reflections

Completeness to theta $=24.96^{\circ}$

Absorption correction

Refinement method

Data / restraints / parameters

Goodness-of-fit on $\mathrm{F}^{2}$

Final R indices [I $>2 \operatorname{sigma}(\mathrm{I})]$

$\mathrm{R}$ indices (all data)

Largest diff. peak and hole 3b

C16 H18 N4 O2

298.34

293(2) K

$0.71073 \AA$

Monoclinic

$\mathrm{C} 2 / \mathrm{c}$

$\mathrm{a}=29.146(6) \AA$

$\alpha=90^{\circ}$.

$\mathrm{b}=5.4240(11) \AA$

$\beta=99.29(3)^{\circ}$.

$\mathrm{c}=9.852(2) \AA$

$\gamma=90^{\circ}$.
1537.1(5) $\AA^{3}$

4

$1.289 \mathrm{Mg} / \mathrm{m}^{3}$

$0.088 \mathrm{~mm}^{-1}$

632

$0.20 \times 0.15 \times 0.10 \mathrm{~mm}^{3}$

1.42 to $24.96^{\circ}$.

$0<=\mathrm{h}<=34,0<=\mathrm{k}<=6,-11<=\mathrm{l}<=11$

1392

$1364[\mathrm{R}(\mathrm{int})=0.0064]$

$100.0 \%$

None

Full-matrix least-squares on $\mathrm{F}^{2}$

1364 / 0 / 100

1.142

$\mathrm{R} 1=0.0451, \mathrm{wR} 2=0.1004$

$\mathrm{R} 1=0.0825, \mathrm{wR} 2=0.1340$

0.161 and -0.255 e. $\AA^{-3}$ 
Table 2. Atomic coordinates ( $\left.\mathrm{x} 10^{4}\right)$ and equivalent isotropic displacement parameters $\left(\AA^{2} \times 10^{3}\right)$ for $3 \mathbf{b}$. $U(e q)$ is defined as one third of the trace of the orthogonalized $U^{\mathrm{ij}}$ tensor.

\begin{tabular}{lrrrr}
\hline & $\mathrm{x}$ & $\mathrm{y}$ & $\mathrm{z}$ & $\mathrm{U}(\mathrm{eq})$ \\
\hline $\mathrm{N}(11)$ & $2002(1)$ & $-2365(5)$ & $11641(2)$ & $67(1)$ \\
$\mathrm{N}(12)$ & $740(1)$ & $951(4)$ & $9840(2)$ & $51(1)$ \\
$\mathrm{O}(10)$ & $828(1)$ & $-664(4)$ & $7807(2)$ & $68(1)$ \\
$\mathrm{C}(10)$ & $1374(1)$ & $-1887(4)$ & $9751(2)$ & $46(1)$ \\
$\mathrm{C}(11)$ & $1627(1)$ & $-1178(5)$ & $11009(2)$ & $56(1)$ \\
$\mathrm{C}(12)$ & $2136(1)$ & $-4343(6)$ & $11007(3)$ & $72(1)$ \\
$\mathrm{C}(13)$ & $1917(1)$ & $-5166(6)$ & $9745(3)$ & $72(1)$ \\
$\mathrm{C}(14)$ & $1529(1)$ & $-3908(5)$ & $9114(3)$ & $61(1)$ \\
$\mathrm{C}(15)$ & $961(1)$ & $-491(5)$ & $9065(2)$ & $47(1)$ \\
$\mathrm{C}(16)$ & $350(1)$ & $2482(5)$ & $9251(2)$ & $53(1)$ \\
$\mathrm{C}(17)$ & $204(1)$ & $4190(5)$ & $10308(2)$ & $52(1)$ \\
& & & & \\
\hline
\end{tabular}

Table 3. Bond lengths $[\AA ̊ \cap]$ and angles $\left[^{\circ}\right]$ for $\mathbf{3 b}$.

\begin{tabular}{llll}
\hline $\mathrm{N}(11)-\mathrm{C}(12)$ & $1.331(4)$ & $\mathrm{C}(15)-\mathrm{N}(12)-\mathrm{C}(16)$ & $121.87(18)$ \\
$\mathrm{N}(11)-\mathrm{C}(11)$ & $1.333(3)$ & $\mathrm{C}(14)-\mathrm{C}(10)-\mathrm{C}(11)$ & $117.4(2)$ \\
$\mathrm{N}(12)-\mathrm{C}(15)$ & $1.331(3)$ & $\mathrm{C}(14)-\mathrm{C}(10)-\mathrm{C}(15)$ & $120.0(2)$ \\
$\mathrm{N}(12)-\mathrm{C}(16)$ & $1.450(3)$ & $\mathrm{C}(11)-\mathrm{C}(10)-\mathrm{C}(15)$ & $122.5(2)$ \\
$\mathrm{O}(10)-\mathrm{C}(15)$ & $1.241(2)$ & $\mathrm{N}(11)-\mathrm{C}(11)-\mathrm{C}(10)$ & $124.1(2)$ \\
$\mathrm{C}(10)-\mathrm{C}(14)$ & $1.376(3)$ & $\mathrm{N}(11)-\mathrm{C}(12)-\mathrm{C}(13)$ & $123.8(3)$ \\
$\mathrm{C}(10)-\mathrm{C}(11)$ & $1.391(3)$ & $\mathrm{C}(12)-\mathrm{C}(13)-\mathrm{C}(14)$ & $118.3(3)$ \\
$\mathrm{C}(10)-\mathrm{C}(15)$ & $1.488(3)$ & $\mathrm{C}(10)-\mathrm{C}(14)-\mathrm{C}(13)$ & $119.7(2)$ \\
$\mathrm{C}(12)-\mathrm{C}(13)$ & $1.378(4)$ & $\mathrm{O}(10)-\mathrm{C}(15)-\mathrm{N}(12)$ & $121.0(2)$ \\
$\mathrm{C}(13)-\mathrm{C}(14)$ & $1.378(4)$ & $\mathrm{O}(10)-\mathrm{C}(15)-\mathrm{C}(10)$ & $121.0(2)$ \\
$\mathrm{C}(16)-\mathrm{C}(17)$ & $1.505(3)$ & $\mathrm{N}(12)-\mathrm{C}(15)-\mathrm{C}(10)$ & $118.04(18)$ \\
$\mathrm{C}(17)-\mathrm{C}(17) \# 1$ & $1.524(5)$ & $\mathrm{N}(12)-\mathrm{C}(16)-\mathrm{C}(17)$ & $111.47(18)$ \\
& & $\mathrm{C}(16)-\mathrm{C}(17)-\mathrm{C}(17) \# 1$ & $111.6(2)$ \\
$\mathrm{C}(12)-\mathrm{N}(11)-\mathrm{C}(11)$ & $116.7(2)$ &
\end{tabular}


Table 4. Anisotropic displacement parameters $\left(\AA^{2} \times 10^{3}\right)$ for $3 \mathbf{b}$. The anisotropic displacement factor exponent takes the form: $-2 \pi^{2}\left[h^{2} a^{* 2} U^{11}+\ldots+2 h k a^{*} b^{*} U^{12}\right]$

\begin{tabular}{lcccccc}
\hline & $\mathrm{U}^{11}$ & $\mathrm{U}^{22}$ & $\mathrm{U}^{33}$ & $\mathrm{U}^{23}$ & $\mathrm{U}^{13}$ & $\mathrm{U}^{12}$ \\
\hline $\mathrm{N}(11)$ & $62(1)$ & $82(2)$ & $52(1)$ & $2(1)$ & $-5(1)$ & $8(1)$ \\
$\mathrm{N}(12)$ & $61(1)$ & $67(1)$ & $24(1)$ & $0(1)$ & $2(1)$ & $15(1)$ \\
$\mathrm{O}(10)$ & $74(1)$ & $101(2)$ & $25(1)$ & $-8(1)$ & $-1(1)$ & $19(1)$ \\
$\mathrm{C}(10)$ & $48(1)$ & $54(1)$ & $35(1)$ & $0(1)$ & $7(1)$ & $1(1)$ \\
$\mathrm{C}(11)$ & $57(1)$ & $68(2)$ & $40(1)$ & $-2(1)$ & $1(1)$ & $7(1)$ \\
$\mathrm{C}(12)$ & $65(2)$ & $84(2)$ & $64(2)$ & $14(2)$ & $4(1)$ & $20(2)$ \\
$\mathrm{C}(13)$ & $80(2)$ & $73(2)$ & $64(2)$ & $-5(2)$ & $10(1)$ & $22(2)$ \\
$\mathrm{C}(14)$ & $68(2)$ & $66(2)$ & $47(1)$ & $-5(1)$ & $8(1)$ & $9(1)$ \\
$\mathrm{C}(15)$ & $52(1)$ & $58(1)$ & $30(1)$ & $1(1)$ & $6(1)$ & $0(1)$ \\
$\mathrm{C}(16)$ & $62(1)$ & $63(2)$ & $32(1)$ & $5(1)$ & $2(1)$ & $13(1)$ \\
$\mathrm{C}(17)$ & $61(1)$ & $56(2)$ & $36(1)$ & $1(1)$ & $2(1)$ & $7(1)$ \\
& & & & & & \\
\hline
\end{tabular}

Table 5. Hydrogen coordinates ( $\left.\mathrm{x} 10^{4}\right)$ and isotropic displacement parameters $\left(\AA^{2} \mathrm{x} 10^{3}\right)$ for $\mathbf{3 b}$.

\begin{tabular}{lrrrr}
\hline & $x$ & $y$ & $z$ & $U(e q)$ \\
& & & & \\
$H(12)$ & 830 & 976 & 10715 & 61 \\
$H(11)$ & 1529 & 210 & 11436 & 67 \\
$\mathrm{H}(12 \mathrm{~A})$ & 2393 & -5221 & 11441 & 86 \\
$\mathrm{H}(13)$ & 2027 & -6536 & 9329 & 87 \\
$\mathrm{H}(14)$ & 1374 & -4422 & 8261 & 73 \\
$\mathrm{H}(16 \mathrm{~A})$ & 90 & 1437 & 8878 & 63 \\
$\mathrm{H}(16 \mathrm{~B})$ & 436 & 3446 & 8502 & 63 \\
$\mathrm{H}(17 \mathrm{~A})$ & 465 & 5227 & 10685 & 62 \\
$\mathrm{H}(17 \mathrm{~B})$ & 117 & 3224 & 11055 & 62 \\
& & & & \\
\hline
\end{tabular}


Table 1. Crystal data and structure refinement for $\mathbf{3 c}$.

Identification code

Empirical formula

Formula weight

Temperature

Wavelength

Crystal system

Space group

Unit cell dimensions

Volume

$\mathrm{Z}$

Density (calculated)

Absorption coefficient

$\mathrm{F}(000)$

Crystal size

Theta range for data collection

Index ranges

Reflections collected

Independent reflections

Completeness to theta $=24.92^{\circ}$

Absorption correction

Refinement method

Data / restraints / parameters

Goodness-of-fit on $\mathrm{F}^{2}$

Final $\mathrm{R}$ indices [I $>2 \operatorname{sigma}(\mathrm{I})]$

$\mathrm{R}$ indices (all data)

Extinction coefficient

Largest diff. peak and hole
$3 c$

C18 H22 N4 O2

326.40

293(2) K

$0.70930 \AA$

Monoclinic

$\mathrm{P} 2(1) / \mathrm{c}$

$\mathrm{a}=5.2230(10) \AA$

$\alpha=90^{\circ}$.

$\mathrm{b}=5.0990(10) \AA$

$\beta=95.19(3)^{\circ}$.

$\mathrm{c}=31.065(6) \AA$

$\gamma=90^{\circ}$.

823.9(3) $\AA^{3}$

2

$1.316 \mathrm{Mg} / \mathrm{m}^{3}$

$0.088 \mathrm{~mm}^{-1}$

348

$0.15 \times 0.10 \times 0.10 \mathrm{~mm}^{3}$

1.31 to $24.92^{\circ}$.

$0<=\mathrm{h}<=6,-6<=\mathrm{k}<=0,-36<=\mathrm{l}<=36$

1556

$1393[\mathrm{R}($ int $)=0.0420]$

$96.5 \%$

None

Full-matrix least-squares on $\mathrm{F}^{2}$

1393 / 0 / 110

1.069

$\mathrm{R} 1=0.0683, \mathrm{wR} 2=0.1276$

$\mathrm{R} 1=0.1769, \mathrm{wR} 2=0.1807$

$0.018(4)$

0.266 and -0.211 e. $\AA^{-3}$ 
Table 2. Atomic coordinates ( $\mathrm{x} 10^{4}$ ) and equivalent isotropic displacement parameters $\left(\AA^{2} \times 10^{3}\right.$ ) for 3c. $U(e q)$ is defined as one third of the trace of the orthogonalized $U^{i j}$ tensor.

\begin{tabular}{lcccc}
\hline & $\mathrm{x}$ & $\mathrm{y}$ & $\mathrm{z}$ & $\mathrm{U}(\mathrm{eq})$ \\
\hline $\mathrm{O}(15)$ & $2302(6)$ & $8168(6)$ & $1021(1)$ & $62(1)$ \\
$\mathrm{N}(10)$ & $8438(7)$ & $9951(8)$ & $1920(1)$ & $62(1)$ \\
$\mathrm{C}(10)$ & $4494(8)$ & $11224(8)$ & $1503(1)$ & $41(1)$ \\
$\mathrm{C}(11)$ & $6600(9)$ & $9606(9)$ & $1592(1)$ & $51(1)$ \\
$\mathrm{C}(12)$ & $8136(10)$ & $12040(10)$ & $2169(2)$ & $63(2)$ \\
$\mathrm{C}(13)$ & $6138(9)$ & $13756(9)$ & $2106(2)$ & $56(2)$ \\
$\mathrm{C}(14)$ & $4286(8)$ & $13355(9)$ & $1768(1)$ & $50(1)$ \\
$\mathrm{C}(15)$ & $2574(9)$ & $10453(8)$ & $1140(1)$ & $45(1)$ \\
$\mathrm{N}(20)$ & $1177(6)$ & $12406(7)$ & $950(1)$ & $49(1)$ \\
$\mathrm{C}(20)$ & $-776(8)$ & $11894(8)$ & $595(1)$ & $47(1)$ \\
$\mathrm{C}(21)$ & $-2299(8)$ & $14308(8)$ & $458(1)$ & $45(1)$ \\
$\mathrm{C}(22)$ & $-4272(8)$ & $13788(8)$ & $78(1)$ & $47(1)$ \\
& & & & \\
\hline
\end{tabular}

Table 3. Bond lengths $[\AA]$ and angles $\left[^{\circ}\right]$ for $\mathbf{3 c}$.

\begin{tabular}{lrll}
\hline $\mathrm{O}(15)-\mathrm{C}(15)$ & $1.227(5)$ & $\mathrm{C}(14)-\mathrm{C}(10)-\mathrm{C}(11)$ & $117.2(4)$ \\
$\mathrm{N}(10)-\mathrm{C}(12)$ & $1.334(6)$ & $\mathrm{C}(14)-\mathrm{C}(10)-\mathrm{C}(15)$ & $125.2(4)$ \\
$\mathrm{N}(10)-\mathrm{C}(11)$ & $1.347(5)$ & $\mathrm{C}(11)-\mathrm{C}(10)-\mathrm{C}(15)$ & $117.6(4)$ \\
$\mathrm{C}(10)-\mathrm{C}(14)$ & $1.374(6)$ & $\mathrm{N}(10)-\mathrm{C}(11)-\mathrm{C}(10)$ & $125.0(4)$ \\
$\mathrm{C}(10)-\mathrm{C}(11)$ & $1.383(6)$ & $\mathrm{N}(10)-\mathrm{C}(12)-\mathrm{C}(13)$ & $123.9(4)$ \\
$\mathrm{C}(10)-\mathrm{C}(15)$ & $1.491(5)$ & $\mathrm{C}(12)-\mathrm{C}(13)-\mathrm{C}(14)$ & $119.4(4)$ \\
$\mathrm{C}(12)-\mathrm{C}(13)$ & $1.362(6)$ & $\mathrm{C}(10)-\mathrm{C}(14)-\mathrm{C}(13)$ & $119.1(4)$ \\
$\mathrm{C}(13)-\mathrm{C}(14)$ & $1.377(5)$ & $\mathrm{O}(15)-\mathrm{C}(15)-\mathrm{N}(20)$ & $121.9(4)$ \\
$\mathrm{C}(15)-\mathrm{N}(20)$ & $1.340(5)$ & $\mathrm{O}(15)-\mathrm{C}(15)-\mathrm{C}(10)$ & $122.0(4)$ \\
$\mathrm{N}(20)-\mathrm{C}(20)$ & $1.458(5)$ & $\mathrm{N}(20)-\mathrm{C}(15)-\mathrm{C}(10)$ & $116.1(4)$ \\
$\mathrm{C}(20)-\mathrm{C}(21)$ & $1.506(5)$ & $\mathrm{C}(15)-\mathrm{N}(20)-\mathrm{C}(20)$ & $121.1(3)$ \\
$\mathrm{C}(21)-\mathrm{C}(22)$ & $1.519(5)$ & $\mathrm{N}(20)-\mathrm{C}(20)-\mathrm{C}(21)$ & $112.5(3)$ \\
$\mathrm{C}(22)-\mathrm{C}(22) \# 1$ & $1.508(8)$ & $\mathrm{C}(20)-\mathrm{C}(21)-\mathrm{C}(22)$ & $112.4(3)$ \\
$\mathrm{C}(12)-\mathrm{N}(10)-\mathrm{C}(11)$ & $115.4(4)$ & $\mathrm{C}(22) \# 1-\mathrm{C}(22)-\mathrm{C}(21)$ & $113.2(4)$
\end{tabular}

Symmetry transformations used to generate equivalent atoms: \#1 -x-1,-y+3,-z 
Table 4. Anisotropic displacement parameters $\left(\AA^{2} \times 10^{3}\right)$ for 3c. The anisotropic displacement factor exponent takes the form: $-2 \pi^{2}\left[h^{2} a^{* 2} U^{11}+\ldots+2 h k a^{*} b^{*} U^{12}\right]$

\begin{tabular}{lllllll}
\hline & $\mathrm{U}^{11}$ & $\mathrm{U}^{22}$ & $\mathrm{U}^{33}$ & $\mathrm{U}^{23}$ & $\mathrm{U}^{13}$ & $\mathrm{U}^{12}$ \\
\hline $\mathrm{O}(15)$ & $70(2)$ & $35(2)$ & $75(2)$ & $-11(2)$ & $-30(2)$ & $4(2)$ \\
$\mathrm{N}(10)$ & $50(3)$ & $61(3)$ & $69(3)$ & $-2(2)$ & $-25(2)$ & $1(2)$ \\
$\mathrm{C}(10)$ & $38(2)$ & $34(2)$ & $49(3)$ & $3(2)$ & $-10(2)$ & $4(2)$ \\
$\mathrm{C}(11)$ & $54(3)$ & $42(3)$ & $54(3)$ & $-4(2)$ & $-12(2)$ & $4(2)$ \\
$\mathrm{C}(12)$ & $65(4)$ & $49(3)$ & $69(4)$ & $5(3)$ & $-29(3)$ & $-12(3)$ \\
$\mathrm{C}(13)$ & $69(3)$ & $39(3)$ & $57(3)$ & $-5(2)$ & $-13(3)$ & $-7(3)$ \\
$\mathrm{C}(14)$ & $47(3)$ & $38(3)$ & $60(3)$ & $1(2)$ & $-10(2)$ & $0(2)$ \\
$\mathrm{C}(15)$ & $51(3)$ & $32(2)$ & $49(3)$ & $1(2)$ & $-9(2)$ & $0(2)$ \\
$\mathrm{N}(20)$ & $52(2)$ & $32(2)$ & $59(2)$ & $-1(2)$ & $-22(2)$ & $-1(2)$ \\
$\mathrm{C}(20)$ & $49(3)$ & $35(2)$ & $54(3)$ & $-4(2)$ & $-18(2)$ & $3(2)$ \\
$\mathrm{C}(21)$ & $41(3)$ & $38(2)$ & $53(3)$ & $0(2)$ & $-11(2)$ & $8(2)$ \\
$\mathrm{C}(22)$ & $42(3)$ & $45(3)$ & $50(3)$ & $-5(2)$ & $-13(2)$ & $3(2)$ \\
& & & & & & \\
\hline
\end{tabular}

Table 5. Hydrogen coordinates ( x 104) and isotropic displacement parameters $\left(\AA^{2} \times 10^{3}\right)$ for $3 c$.

\begin{tabular}{lrrrr}
\hline & $x$ & $y$ & $z$ & $U(e q)$ \\
& & & & \\
$H(11)$ & 6760 & 8171 & 1411 & 61 \\
$H(12)$ & 9364 & 12343 & 2400 & 76 \\
$H(13)$ & 6026 & 15184 & 2290 & 67 \\
$H(14)$ & 2913 & 14511 & 1720 & 59 \\
$H(20)$ & 1437 & 13989 & 1039 & 59 \\
$H(20 A)$ & -1940 & 10552 & 682 & 57 \\
$H(20 B)$ & 49 & 11225 & 349 & 57 \\
$H(21 A)$ & -1131 & 15670 & 380 & 54 \\
$H(21 B)$ & -3173 & 14942 & 700 & 54 \\
$H(22 A)$ & -3404 & 13066 & -159 & 56 \\
$H(22 B)$ & -5485 & 12485 & 162 & 56 \\
& & & & \\
\hline
\end{tabular}


Table 1. Crystal data and structure refinement for $\mathbf{3 d}$.

Identification code

Empirical formula

Formula weight

Temperature

Wavelength

Crystal system

Space group

Unit cell dimensions

Volume

$\mathrm{Z}$

Density (calculated)

Absorption coefficient

$\mathrm{F}(000)$

Crystal size

Theta range for data collection

Index ranges

Reflections collected

Independent reflections

Completeness to theta $=24.97^{\circ}$

Absorption correction

Refinement method

Data / restraints / parameters

Goodness-of-fit on $\mathrm{F}^{2}$

Final $\mathrm{R}$ indices [I $>2 \operatorname{sigma}(\mathrm{I})]$

$\mathrm{R}$ indices (all data)

Extinction coefficient

Largest diff. peak and hole 3d

C20 H26 N4 O2

354.45

293(2) K

$0.71073 \AA$

Monoclinic

$\mathrm{P} 2(1) / \mathrm{c}$

$\mathrm{a}=5.2420(10) \AA$

$\alpha=90^{\circ}$.

$\mathrm{b}=5.1020(10) \AA$

$\beta=90.73(3)^{\circ}$.

$\mathrm{c}=34.127(7) \AA$

912.6(3) $\AA^{3}$

2

$1.290 \mathrm{Mg} / \mathrm{m}^{3}$

$0.085 \mathrm{~mm}^{-1}$

380

$0.15 \times 0.10 \times 0.10 \mathrm{~mm}^{3}$

1.19 to $24.97^{\circ}$.

$0<=\mathrm{h}<=6,0<=\mathrm{k}<=6,-40<=1<=40$

1731

$1547[\mathrm{R}($ int $)=0.0369]$

$96.9 \%$

None

Full-matrix least-squares on $\mathrm{F}^{2}$

1547 / 0 / 119

1.065

$\mathrm{R} 1=0.0505, \mathrm{wR} 2=0.1121$

$\mathrm{R} 1=0.1055, \mathrm{wR} 2=0.1540$

$0.007(2)$

0.194 and -0.175 e. $\AA^{-3}$ 
Table 2. Atomic coordinates ( $\left.\mathrm{x} 10^{4}\right)$ and equivalent isotropic displacement parameters $\left(\AA^{2} \times 10^{3}\right)$ for 3d. $U(e q)$ is defined as one third of the trace of the orthogonalized $U^{i j}$ tensor.

\begin{tabular}{lrrrr}
\hline & $\mathrm{x}$ & $\mathrm{y}$ & $\mathrm{z}$ & $\mathrm{U}(\mathrm{eq})$ \\
\hline $\mathrm{O}(10)$ & $1815(5)$ & $2231(4)$ & $1170(1)$ & $56(1)$ \\
$\mathrm{N}(10)$ & $-3785(5)$ & $4014(6)$ & $1976(1)$ & $59(1)$ \\
$\mathrm{C}(10)$ & $-101(5)$ & $5245(6)$ & $1605(1)$ & $39(1)$ \\
$\mathrm{C}(11)$ & $-2144(6)$ & $3642(6)$ & $1685(1)$ & $48(1)$ \\
$\mathrm{C}(12)$ & $-3341(6)$ & $6090(7)$ & $2201(1)$ & $57(1)$ \\
$\mathrm{C}(13)$ & $-1373(7)$ & $7805(7)$ & $2147(1)$ & $57(1)$ \\
$\mathrm{C}(14)$ & $298(6)$ & $7395(6)$ & $1842(1)$ & $47(1)$ \\
$\mathrm{C}(15)$ & $1619(6)$ & $4530(6)$ & $1275(1)$ & $44(1)$ \\
$\mathrm{N}(20)$ & $2884(5)$ & $6452(5)$ & $1103(1)$ & $45(1)$ \\
$\mathrm{C}(20)$ & $4636(6)$ & $5928(6)$ & $783(1)$ & $44(1)$ \\
$\mathrm{C}(21)$ & $6040(6)$ & $8346(6)$ & $663(1)$ & $42(1)$ \\
$\mathrm{C}(22)$ & $7790(5)$ & $7841(6)$ & $318(1)$ & $44(1)$ \\
$\mathrm{C}(23)$ & $9134(6)$ & $10261(6)$ & $173(1)$ & $44(1)$ \\
& & & & \\
\hline
\end{tabular}

Table 3. Bond lengths $[\AA]$ and angles $\left[{ }^{\circ}\right]$ for $\mathbf{3 d}$.

\begin{tabular}{llll}
\hline $\mathrm{O}(10)-\mathrm{C}(15)$ & $1.231(4)$ & & \\
$\mathrm{N}(10)-\mathrm{C}(12)$ & $1.327(4)$ & $\mathrm{C}(12)-\mathrm{N}(10)-\mathrm{C}(11)$ & $115.7(3)$ \\
$\mathrm{N}(10)-\mathrm{C}(11)$ & $1.335(4)$ & $\mathrm{C}(11)-\mathrm{C}(10)-\mathrm{C}(14)$ & $118.0(3)$ \\
$\mathrm{C}(10)-\mathrm{C}(11)$ & $1.377(4)$ & $\mathrm{C}(11)-\mathrm{C}(10)-\mathrm{C}(15)$ & $118.7(3)$ \\
$\mathrm{C}(10)-\mathrm{C}(14)$ & $1.377(4)$ & $\mathrm{C}(14)-\mathrm{C}(10)-\mathrm{C}(15)$ & $123.2(3)$ \\
$\mathrm{C}(10)-\mathrm{C}(15)$ & $1.498(4)$ & $\mathrm{N}(10)-\mathrm{C}(11)-\mathrm{C}(10)$ & $124.9(3)$ \\
$\mathrm{C}(12)-\mathrm{C}(13)$ & $1.367(5)$ & $\mathrm{N}(10)-\mathrm{C}(12)-\mathrm{C}(13)$ & $124.1(3)$ \\
$\mathrm{C}(13)-\mathrm{C}(14)$ & $1.384(4)$ & $\mathrm{C}(12)-\mathrm{C}(13)-\mathrm{C}(14)$ & $119.3(3)$ \\
$\mathrm{C}(15)-\mathrm{N}(20)$ & $1.325(4)$ & $\mathrm{C}(10)-\mathrm{C}(14)-\mathrm{C}(13)$ & $117.9(3)$ \\
$\mathrm{N}(20)-\mathrm{C}(20)$ & $1.460(3)$ & $\mathrm{O}(10)-\mathrm{C}(15)-\mathrm{N}(20)$ & $122.2(3)$ \\
$\mathrm{C}(20)-\mathrm{C}(21)$ & $1.497(4)$ & $\mathrm{O}(10)-\mathrm{C}(15)-\mathrm{C}(10)$ & $120.3(3)$ \\
$\mathrm{C}(21)-\mathrm{C}(22)$ & $1.522(4)$ & $\mathrm{N}(20)-\mathrm{C}(15)-\mathrm{C}(10)$ & $117.5(3)$ \\
$\mathrm{C}(22)-\mathrm{C}(23)$ & $1.508(4)$ & $\mathrm{C}(15)-\mathrm{N}(20)-\mathrm{C}(20)$ & $121.2(2)$ \\
$\mathrm{C}(23)-\mathrm{C}(23) \# 1$ & $1.522(5)$ & $\mathrm{N}(20)-\mathrm{C}(20)-\mathrm{C}(21)$ & $111.8(2)$
\end{tabular}




$\begin{array}{llll}\mathrm{C}(20)-\mathrm{C}(21)-\mathrm{C}(22) & 112.1(2) & \mathrm{C}(22)-\mathrm{C}(23)-\mathrm{C}(23) \# 1 & 113.4(3) \\ \mathrm{C}(23)-\mathrm{C}(22)-\mathrm{C}(21) & 113.8(2) & \end{array}$

Symmetry transformations used to generate equivalent atoms:

$\# 1-x+2,-y+2,-z$

Table 4. Anisotropic displacement parameters $\left(\AA^{2} \times 10^{3}\right)$ for 3d. The anisotropic displacement factor exponent takes the form: $-2 \pi^{2}\left[h^{2} a^{* 2} U^{11}+\ldots+2 h k a^{*} b^{*} U^{12}\right]$

\begin{tabular}{lllllll}
\hline & $\mathrm{U}^{11}$ & $\mathrm{U}^{22}$ & $\mathrm{U}^{33}$ & $\mathrm{U}^{23}$ & $\mathrm{U}^{13}$ & $\mathrm{U}^{12}$ \\
\hline $\mathrm{O}(10)$ & $71(2)$ & $34(1)$ & $65(2)$ & $-7(1)$ & $30(1)$ & $-6(1)$ \\
$\mathrm{N}(10)$ & $57(2)$ & $57(2)$ & $64(2)$ & $-1(2)$ & $26(2)$ & $-2(2)$ \\
$\mathrm{C}(10)$ & $42(2)$ & $37(2)$ & $39(2)$ & $5(1)$ & $11(1)$ & $4(1)$ \\
$\mathrm{C}(11)$ & $52(2)$ & $41(2)$ & $52(2)$ & $2(2)$ & $14(2)$ & $-1(2)$ \\
$\mathrm{C}(12)$ & $58(2)$ & $58(2)$ & $56(2)$ & $6(2)$ & $28(2)$ & $12(2)$ \\
$\mathrm{C}(13)$ & $74(2)$ & $51(2)$ & $46(2)$ & $-2(2)$ & $13(2)$ & $11(2)$ \\
$\mathrm{C}(14)$ & $50(2)$ & $41(2)$ & $50(2)$ & $-3(1)$ & $14(2)$ & $-4(2)$ \\
$\mathrm{C}(15)$ & $47(2)$ & $37(2)$ & $47(2)$ & $1(1)$ & $10(1)$ & $0(1)$ \\
$\mathrm{N}(20)$ & $55(2)$ & $32(1)$ & $48(2)$ & $1(1)$ & $19(1)$ & $2(1)$ \\
$\mathrm{C}(20)$ & $47(2)$ & $39(2)$ & $48(2)$ & $-3(1)$ & $23(1)$ & $-3(1)$ \\
$\mathrm{C}(21)$ & $47(2)$ & $35(2)$ & $45(2)$ & $-1(1)$ & $14(1)$ & $0(1)$ \\
$\mathrm{C}(22)$ & $44(2)$ & $42(2)$ & $45(2)$ & $-6(1)$ & $15(1)$ & $-1(1)$ \\
$\mathrm{C}(23)$ & $43(2)$ & $38(2)$ & $52(2)$ & $-2(1)$ & $16(1)$ & $-5(1)$ \\
& & & & & & \\
\hline
\end{tabular}


Table 5. Hydrogen coordinates ( $\mathrm{x} 10^{4}$ ) and isotropic displacement parameters $\left(\AA^{2} \times 10^{3}\right)$ for $3 d$.

\begin{tabular}{lrrrr}
\hline & $x$ & $y$ & $z$ & $U(e q)$ \\
& & & & \\
$H(11)$ & -2399 & 2192 & 1524 & 58 \\
$H(12)$ & -4438 & 6397 & 2408 & 68 \\
$\mathrm{H}(13)$ & -1160 & 9234 & 2313 & 68 \\
$\mathrm{H}(14)$ & 1649 & 8536 & 1799 & 56 \\
$\mathrm{H}(20)$ & 2670 & 8038 & 1182 & 54 \\
$\mathrm{H}(20 \mathrm{~A})$ & 3682 & 5245 & 560 & 53 \\
$\mathrm{H}(20 \mathrm{~B})$ & 5855 & 4602 & 866 & 53 \\
$\mathrm{H}(21 \mathrm{~A})$ & 7047 & 8987 & 883 & 50 \\
$\mathrm{H}(21 \mathrm{~B})$ & 4817 & 9696 & 591 & 50 \\
$\mathrm{H}(22 \mathrm{~A})$ & 9061 & 6553 & 396 & 52 \\
$\mathrm{H}(22 \mathrm{~B})$ & 6790 & 7099 & 104 & 52 \\
$\mathrm{H}(23 \mathrm{~A})$ & 10144 & 10998 & 386 & 53 \\
$\mathrm{H}(23 \mathrm{~B})$ & 7865 & 11554 & 96 & 53 \\
& & & &
\end{tabular}


Table 1. Crystal data and structure refinement for $\mathbf{3 e}$.

Identification code

Empirical formula

Formula weight

Temperature

Wavelength

Crystal system

Space group

Unit cell dimensions

Volume

Z

Density (calculated)

Absorption coefficient

$\mathrm{F}(000)$

Crystal size

Theta range for data collection

Index ranges

Reflections collected

Independent reflections

Completeness to theta $=24.98^{\circ}$

Absorption correction

Refinement method

Data / restraints / parameters

Goodness-of-fit on $\mathrm{F}^{2}$

Final $\mathrm{R}$ indices $[\mathrm{I}>2 \operatorname{sigma}(\mathrm{I})]$

$\mathrm{R}$ indices (all data)

Extinction coefficient

Largest diff. peak and hole 3e

C18 H14 N4 O2

318.33

293(2) K

$0.71073 \AA$

Triclinic

P-1

$\mathrm{a}=5.1940(10) \AA$

$\alpha=94.82(3)^{\circ}$.

$\mathrm{b}=6.9260(14) \AA$

$\beta=102.00(3)^{\circ}$.

$\mathrm{c}=11.034(2) \AA$

$\gamma=107.64(3)^{\circ}$.

365.37(15) $\AA^{3}$

1

$1.447 \mathrm{Mg} / \mathrm{m}^{3}$

$0.098 \mathrm{~mm}^{-1}$

166

$0.20 \times 0.15 \times 0.10 \mathrm{~mm}^{3}$

1.91 to $24.98^{\circ}$.

$0<=\mathrm{h}<=6,-8<=\mathrm{k}<=7,-13<=\mathrm{l}<=12$

1434

$1282[\mathrm{R}(\mathrm{int})=0.0106]$

$99.9 \%$

None

Full-matrix least-squares on $\mathrm{F}^{2}$

1282 / 0 / 110

1.084

$\mathrm{R} 1=0.0350, \mathrm{wR} 2=0.0906$

$\mathrm{R} 1=0.0451, \mathrm{wR} 2=0.1014$

$0.140(15)$

0.194 and -0.168 e. $\AA^{-3}$ 
Table 2. Atomic coordinates ( $\left.\mathrm{x} 10^{4}\right)$ and equivalent isotropic displacement parameters $\left(\AA^{2} \times 10^{3}\right)$ for 3e. $U(e q)$ is defined as one third of the trace of the orthogonalized $U^{i j}$ tensor.

\begin{tabular}{lrrrr}
\hline & $\mathrm{x}$ & $\mathrm{y}$ & $\mathrm{z}$ & $\mathrm{U}(\mathrm{eq})$ \\
\hline $\mathrm{O}(16)$ & $383(2)$ & $6604(2)$ & $1900(1)$ & $43(1)$ \\
$\mathrm{N}(11)$ & $1334(3)$ & $2748(3)$ & $4479(2)$ & $48(1)$ \\
$\mathrm{N}(12)$ & $4647(3)$ & $6968(2)$ & $1551(1)$ & $32(1)$ \\
$\mathrm{C}(11)$ & $2800(3)$ & $4495(2)$ & $2833(1)$ & $28(1)$ \\
$\mathrm{C}(12)$ & $1400(3)$ & $4225(3)$ & $3776(2)$ & $38(1)$ \\
$\mathrm{C}(13)$ & $2724(4)$ & $1482(3)$ & $4237(2)$ & $43(1)$ \\
$\mathrm{C}(14)$ & $4225(3)$ & $1661(2)$ & $3346(2)$ & $37(1)$ \\
$\mathrm{C}(15)$ & $4283(3)$ & $3195(2)$ & $2626(2)$ & $33(1)$ \\
$\mathrm{C}(16)$ & $2493(3)$ & $6113(2)$ & $2057(1)$ & $29(1)$ \\
$\mathrm{C}(17)$ & $4769(3)$ & $8490(2)$ & $767(1)$ & $28(1)$ \\
$\mathrm{C}(18)$ & $7331(3)$ & $9958(2)$ & $848(1)$ & $32(1)$ \\
$\mathrm{C}(19)$ & $7568(3)$ & $11454(2)$ & $90(1)$ & $32(1)$ \\
& & & & \\
\hline
\end{tabular}

Table 3. Bond lengths $[\AA]$ and angles $\left[{ }^{\circ}\right]$ for $\mathbf{3 e}$.

\begin{tabular}{llll}
\hline $\mathrm{O}(16)-\mathrm{C}(16)$ & $1.2256(18)$ & & \\
$\mathrm{N}(11)-\mathrm{C}(12)$ & $1.331(2)$ & $\mathrm{C}(12)-\mathrm{N}(11)-\mathrm{C}(13)$ & $116.58(14)$ \\
$\mathrm{N}(11)-\mathrm{C}(13)$ & $1.337(2)$ & $\mathrm{C}(16)-\mathrm{N}(12)-\mathrm{C}(17)$ & $125.52(12)$ \\
$\mathrm{N}(12)-\mathrm{C}(16)$ & $1.3521(19)$ & $\mathrm{C}(12)-\mathrm{C}(11)-\mathrm{C}(15)$ & $118.42(14)$ \\
$\mathrm{N}(12)-\mathrm{C}(17)$ & $1.4135(19)$ & $\mathrm{C}(12)-\mathrm{C}(11)-\mathrm{C}(16)$ & $117.51(13)$ \\
$\mathrm{C}(11)-\mathrm{C}(12)$ & $1.382(2)$ & $\mathrm{C}(15)-\mathrm{C}(11)-\mathrm{C}(16)$ & $124.00(13)$ \\
$\mathrm{C}(11)-\mathrm{C}(15)$ & $1.384(2)$ & $\mathrm{N}(11)-\mathrm{C}(12)-\mathrm{C}(11)$ & $123.74(15)$ \\
$\mathrm{C}(11)-\mathrm{C}(16)$ & $1.4927(19)$ & $\mathrm{N}(11)-\mathrm{C}(13)-\mathrm{C}(14)$ & $123.82(15)$ \\
$\mathrm{C}(13)-\mathrm{C}(14)$ & $1.368(2)$ & $\mathrm{C}(13)-\mathrm{C}(14)-\mathrm{C}(15)$ & $119.09(14)$ \\
$\mathrm{C}(14)-\mathrm{C}(15)$ & $1.376(2)$ & $\mathrm{C}(14)-\mathrm{C}(15)-\mathrm{C}(11)$ & $118.31(14)$ \\
$\mathrm{C}(17)-\mathrm{C}(19) \# 1$ & $1.388(2)$ & $\mathrm{O}(16)-\mathrm{C}(16)-\mathrm{N}(12)$ & $123.27(13)$ \\
$\mathrm{C}(17)-\mathrm{C}(18)$ & $1.388(2)$ & $\mathrm{O}(16)-\mathrm{C}(16)-\mathrm{C}(11)$ & $120.63(13)$ \\
$\mathrm{C}(18)-\mathrm{C}(19)$ & $1.379(2)$ & $\mathrm{N}(12)-\mathrm{C}(16)-\mathrm{C}(11)$ & $116.10(12)$ \\
$\mathrm{C}(19)-\mathrm{C}(17) \# 1$ & $1.388(2)$ & $\mathrm{C}(19) \# 1-\mathrm{C}(17)-\mathrm{C}(18)$ & $119.13(14)$
\end{tabular}




$\begin{array}{llll}\mathrm{C}(19) \# 1-\mathrm{C}(17)-\mathrm{N}(12) & 122.33(13) & \mathrm{C}(19)-\mathrm{C}(18)-\mathrm{C}(17) & 120.79(14) \\ \mathrm{C}(18)-\mathrm{C}(17)-\mathrm{N}(12) & 118.54(13) & \mathrm{C}(18)-\mathrm{C}(19)-\mathrm{C}(17) \# 1 & 120.08(14)\end{array}$

Symmetry transformations used to generate equivalent atoms:

$\# 1-\mathrm{x}+1,-\mathrm{y}+2,-\mathrm{z}$

Table 4. Anisotropic displacement parameters $\left(\AA^{2} \times 10^{3}\right)$ for 3e. The anisotropic

displacement factor exponent takes the form: $-2 \pi^{2}\left[h^{2} a^{* 2} U^{11}+\ldots+2 h k a^{*} b^{*} U^{12}\right]$

\begin{tabular}{lcccccc}
\hline & $\mathrm{U}^{11}$ & $\mathrm{U}^{22}$ & $\mathrm{U}^{33}$ & $\mathrm{U}^{23}$ & $\mathrm{U}^{13}$ & $\mathrm{U}^{12}$ \\
\hline $\mathrm{O}(16)$ & $34(1)$ & $50(1)$ & $64(1)$ & $32(1)$ & $25(1)$ & $25(1)$ \\
$\mathrm{N}(11)$ & $50(1)$ & $60(1)$ & $54(1)$ & $35(1)$ & $28(1)$ & $29(1)$ \\
$\mathrm{N}(12)$ & $27(1)$ & $36(1)$ & $45(1)$ & $22(1)$ & $15(1)$ & $17(1)$ \\
$\mathrm{C}(11)$ & $23(1)$ & $28(1)$ & $33(1)$ & $9(1)$ & $6(1)$ & $8(1)$ \\
$\mathrm{C}(12)$ & $37(1)$ & $44(1)$ & $45(1)$ & $19(1)$ & $18(1)$ & $21(1)$ \\
$\mathrm{C}(13)$ & $45(1)$ & $44(1)$ & $50(1)$ & $28(1)$ & $15(1)$ & $20(1)$ \\
$\mathrm{C}(14)$ & $39(1)$ & $33(1)$ & $46(1)$ & $13(1)$ & $9(1)$ & $18(1)$ \\
$\mathrm{C}(15)$ & $33(1)$ & $34(1)$ & $38(1)$ & $12(1)$ & $13(1)$ & $13(1)$ \\
$\mathrm{C}(16)$ & $27(1)$ & $28(1)$ & $36(1)$ & $11(1)$ & $11(1)$ & $11(1)$ \\
$\mathrm{C}(17)$ & $29(1)$ & $29(1)$ & $34(1)$ & $12(1)$ & $13(1)$ & $15(1)$ \\
$\mathrm{C}(18)$ & $25(1)$ & $38(1)$ & $37(1)$ & $15(1)$ & $9(1)$ & $14(1)$ \\
$\mathrm{C}(19)$ & $25(1)$ & $33(1)$ & $38(1)$ & $12(1)$ & $10(1)$ & $8(1)$ \\
& & & & & & \\
\hline
\end{tabular}


Table 5. Hydrogen coordinates ( $\times 10^{4}$ ) and isotropic displacement parameters $\left(\AA^{2} \times 10^{3}\right)$ for $3 \mathbf{e}$.

\begin{tabular}{lrrrr}
\hline & $x$ & $y$ & $z$ & $U(e q)$ \\
& & & & \\
$H(12)$ & 6062 & 6551 & 1720 & 39 \\
$H(12 A)$ & 450 & 5127 & 3928 & 46 \\
$H(13)$ & 2667 & 416 & 4702 & 52 \\
$H(14)$ & 5193 & 758 & 3228 & 45 \\
$H(15)$ & 5293 & 3354 & 2016 & 40 \\
$H(18)$ & 8905 & 9931 & 1421 & 38 \\
$H(19)$ & 9297 & 12428 & 153 & 38 \\
& & & & \\
\hline
\end{tabular}

